This is the pre-peer reviewed version of the following article:

Oscar Francesconi, Cristina Nativi, Gabriele Gabrielli, Irene De Simone, Sam Noppen, Jan Balzarini, Sandra Liekens, Stefano Roelens "Antiviral Activity of Synthetic Aminopyrrolic Carbohydrate Binding Agents: Targeting the Glycans of Viral gp120 to Inhibit HIV Entry" Chem. Eur. J., 2015, 21, 10089 -10093, which has been published in final form at [DOI:10.1002/chem.201501030]. This article may be used for non-commercial purposes in accordance with Wiley Terms and Conditions for Use of Self-Archived Versions 


\title{
Antiviral Activity of Synthetic Aminopyrrolic Carbohydrate Binding Agents. Targeting the Glycans of Viral gp120 to Inhibit HIV Entry.
}

\author{
Oscar Francesconi, ${ }^{[a]}$ Cristina Nativi, ${ }^{[a]}$ Gabriele Gabrielli, ${ }^{[a]}$ Irene De Simone,${ }^{[a]}$ Sam Noppen,${ }^{[b]}$ Jan \\ Balzarini, ${ }^{[b]}$ Sandra Liekens ${ }^{[b]}$ and Stefano Roelens ${ }^{[c]}$
}

\begin{abstract}
The binding abilities of a set of structurally related aminopyrrolic synthetic receptors for mannosides, endowed with antimycotic activity against yeast and yeast-like pathogens bearing mannoproteins on their cell surface, have been investigated towards the highly mannosylated gp120 and gp41 glycoproteins of the HIV envelope. A pronounced binding interaction with both glycoproteins was observed by SPR for most of the investigated compounds. Comparison of their binding properties towards the glycoproteins with their binding affinities toward mannosides revealed a direct correlation, supporting their role as carbohydrate binding agents (CBAs). Cytostatic activity studies revealed antiproliferative activity dependent on the nature and the structure of compounds. Antiviral activity studies against a broad panel of DNA and RNA viruses, showed an inhibitory effect against HIV infection of T-lymphocyte CEM cells for two compounds, suggesting antiviral activity similar to other CBAs, such as the non-peptidic pradimicin antibiotics.
\end{abstract}

\section{Introduction}

The carbohydrates of the glycocalyx, exposed on the surface of eukaryotic cells, are critically involved in the modulation of the immune system and in cell adhesion processes. ${ }^{[1]}$ Many pathogenic microorganisms, including several viruses, are also covered by a glycoconjugate coating, which is used to promote adhesion to and infection of the host cells through carbohydrate recognition processes. ${ }^{[2]}$ The densely mannosylated N-glycans of the viral envelope of the human immunodeficiency virus (HIV) play a crucial role in viral transmission. The glycan coating also acts as a shield for the virus, hiding the underlying immunogenic epitopes in order to escape from the immune system of the

[a] Dr. O. Francesconi, Prof. C. Nativi, Dr. G. Gabrielli, I. De Simone Dipartimento di Chimica, Università di Firenze

Polo Scientifico e Tecnologico,

50019 Sesto Fiorentino, Firenze (Italy)

E-mail: oscar.francesconi@unifi.it

[b] S. Noppen, Prof. J. Balzarini, Prof. S. Liekens

Rega Institute for Medical Research, KU Leuven,

B-3000 Leuven (Belgium)

[C] Dr. S. Roelens

Istituto di Metodologie Chimiche (IMC)

Consiglio Nazionale delle Ricerche (CNR), Dipartimento di Chimica Polo Scientifico e Tecnologico

50019 Sesto Fiorentino, Firenze (Italy)

Supporting information for this article is given via a link at the end of the document.((Please delete this text if not appropriate)) host. ${ }^{[3,4]}$

Recently, the highly mannosylated glycoprotein gp120 of the HIV envelope has been proposed as a target for new therapeutic strategies. ${ }^{[4 a, b]}$ Lectins of various origins, capable of interacting with gp120, turned out to effectively inhibit infection and transmission of HIV by blocking the virus entry process to the host cell. ${ }^{[4, d]}$ For example, microvirin exerts a potent inhibitory activity toward HIV-1 infection $\left(I C_{50}=2-12 \mathrm{nM}\right)$ by selectively recognizing the Man $\alpha(1-2)$ Man motif of the gp120 viral protein. ${ }^{[5 a, b]}$ Moreover, long-term exposure to doseescalating concentrations of lectins causes several mutations in the viral gp120, predominantly affecting the N-glycosylation sites. As a result, the partially deficient glycosylated envelope uncovers immunogenic epitopes, which are thus exposed to the immune system and may trigger a humoral response. ${ }^{[6]} \mathrm{Hu}$ et al. have indeed demonstrated that cyanovirin-exposed HIV-1 strains that had a few deleted N-glycosylation motifs in gp120, were more susceptible to the inhibitory activity of several gp120targeting monoclonal antibodies. ${ }^{[6 b]}$ This dual activity mechanism makes the use of carbohydrate binding agents (CBAs) very attractive.

Unfortunately, lectins present many drawbacks which prevent their development as therapeutic agents, mainly due to their high molecular weight and their peptidic nature. ${ }^{[4 c, d]}$ Therefore the development of small-size nonpeptidic molecules as CBAs is recently emerging as a promising strategy for antiviral therapies and vaccines. For example, the nonpeptidic antibiotic Pradimicin A (PRM-A) and its more soluble analogue Pradimicin S (PRM-S), which have been found to bind to the terminal mannosides exposed on fungal membranes, ${ }^{[7,8]}$ showed antiviral activity against HIV-1, with $50 \%$ effective concentrations $\left(E C_{50}\right.$ 's $)$ in the low micromolar range. ${ }^{[9 a-c]}$ Non-peptidic $1,3,5-$ triazines, shown to bind to gp120 and to inhibit HIV-1 in cell culture, have also been reported, ${ }^{[9 \mathrm{~d}]}$ as well as glycan-binding benzoboroxole-functionalized polymers. ${ }^{\left[{ }^{[e, f]}\right.}$ Thus, the search for new (preferably non-peptidic) CBAs is a topic of growing interest in the current literature.

In the last few years we have developed a family of structurally-related synthetic aminopyrrolic receptors for biomimetic recognition of mono- and disaccharides of biological interest. ${ }^{[10]}$ They were found to bind to mono- and dimannosides in competitive organic solvents with affinities in the low micromolar range by exploiting a combination of hydrogen bonding and van der Waals/CH-m interactions. ${ }^{[11]}$ The progenitor of this family is the tripodal aminopyrrolic receptor 1 whose structure interestingly presents close similarities with the architecture of the primary mannose binding site of PRM-A, as 
<smiles>CCc1c(CNCc2ccc[nH]2)c(CC)c(CNCc2ccc[nH]2)c(CC)c1CNCc1ccc[nH]1</smiles><smiles>c1c[nH]c(CNCc2c(CNCc3ccc[nH]3)c(CNCc3ccc[nH]3)c(CNCc3ccc[nH]3)c(CNCc3ccc[nH]3)c2CNCc2ccc[nH]2)c1</smiles><smiles>CCc1c(CN)c(CC)c(CN)c(CC)c1CN</smiles><smiles>CNCc1cc(CNCc2ccc[nH]2)cc(CNCc2ccc[nH]2)c1</smiles>

3

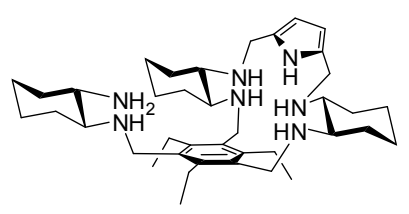

$(S)-8$

$(R)-8$

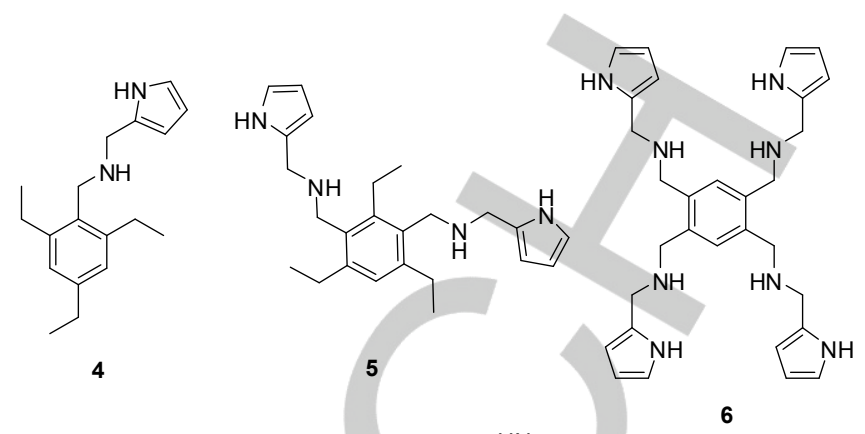

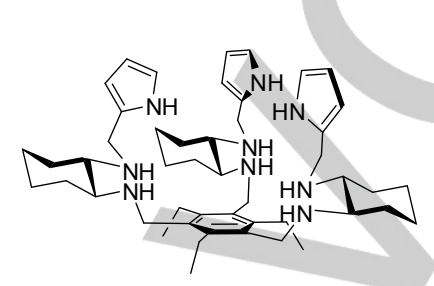

(S)-9

$(R)-9$

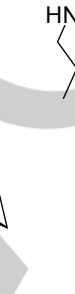

$\stackrel{\mathrm{HN}}{>}\rangle$

Chart 1. Set of the aminopyrrolic compounds investigated in this study.

Table 1. Binding affinities $\left(B C_{50}{ }^{0}, \mathrm{mM}\right)$ of compounds 1 to 10 for octyl mannosides in $\mathrm{CD}_{3} \mathrm{CN}$. ${ }^{[a]}$

\begin{tabular}{|c|c|c|c|c|c|c|c|c|c|c|c|c|}
\hline & 1 & 2 & 3 & 4 & 5 & 6 & 7 & $(S)-8$ & $(R)-8$ & $(S)-\mathbf{9}$ & $(R)-9$ & 10 \\
\hline Oct $\alpha$ Man & 15.6 & n.d. & 22.0 & n.d. & 98.0 & 12.0 & 8.20 & 0.65 & 1.16 & 0.44 & 0.13 & 4.00 \\
\hline Oct $\beta$ Man & 7.40 & n.d. & 20.6 & n.d. & 44.5 & 5.90 & 8.00 & 0.42 & 1.34 & 0.57 & 0.87 & 0.65 \\
\hline
\end{tabular}

[a] Formation constants $(\log \beta)$ were measured by ${ }^{1} \mathrm{H}$ NMR $(400 \mathrm{MHz})$ from titration experiments at $T=298 \mathrm{~K}$. Data from previous works (see ref $11 \mathrm{~b}$ and 13$)$ Intrinsic Median Binding Concentration $\left(B C_{50}{ }^{\circ}\right)$ values were calculated from the $(\log \beta)$ values using the "BC 50 Calculator" Program (see ref. 15).

suggested by Ito and Nakagawa. ${ }^{[12 a, b]}$ In order to evaluate the efficacy of these receptors as CBAs, we have recently tested their biological activity toward several strains of yeast and yeastlike microorganisms, which present a close carbohydrate similarity between the mannan portion exposed on their cell surface and the oligomannose glycans of the gp120 glycoprotein of the viral envelope of HIV. ${ }^{[13]}$ The results obtained against different pathogenic microorganisms, like Pichia norvegensis and Prototheca wickeramii, demonstrated antifungal activity and cytotoxicity comparable to commercial antifungal drugs like Amphotericin B and Ketoconazole. Moreover, localization studies on treated cells suggested that the interaction of these compounds with the surface glycans represents a key step in the internalization into the cell, where they exert their antibiotic activity.

Because of the close similarity of the mannan portion of yeast cell wall with the HIV gp120 glycans, ${ }^{[14]}$ we carried out an investigation on the antiviral activity of this family of synthetic receptors. We report in the present paper the results of such a study on the inhibitory activity of a set of synthetic aminopyrrolic receptors against viruses of high health concern, such as HIV. The cytostatic activities of the investigated compounds were also examined, to be compared to their antiviral activities.

\section{Results and Discussion}

Chemistry. The set of investigated molecules (Chart 1) was obtained from structural modifications of the parent tripodal aminopyrrolic receptor $\mathbf{1}$, which exhibited the most interesting biological activities towards yeasts. The modifications carried out concerned: i) dissection of the architecture into the constituting structural elements (2 and 3 ); ii) variation of the number of aminopyrrolic binding arms on the benzene platform (4, 5, 6 and $7)$; iii) introduction of additional aminic binding groups inserted between the pyrrolic and the benzenic ring $((S)-8,(R)-8,(S)-9$ and $(R)-9)$ or on position 5 of the pyrrole ring (10). All molecules were prepared according to previously described methods. ${ }^{[11 \mathrm{~b}, 13]}$ 

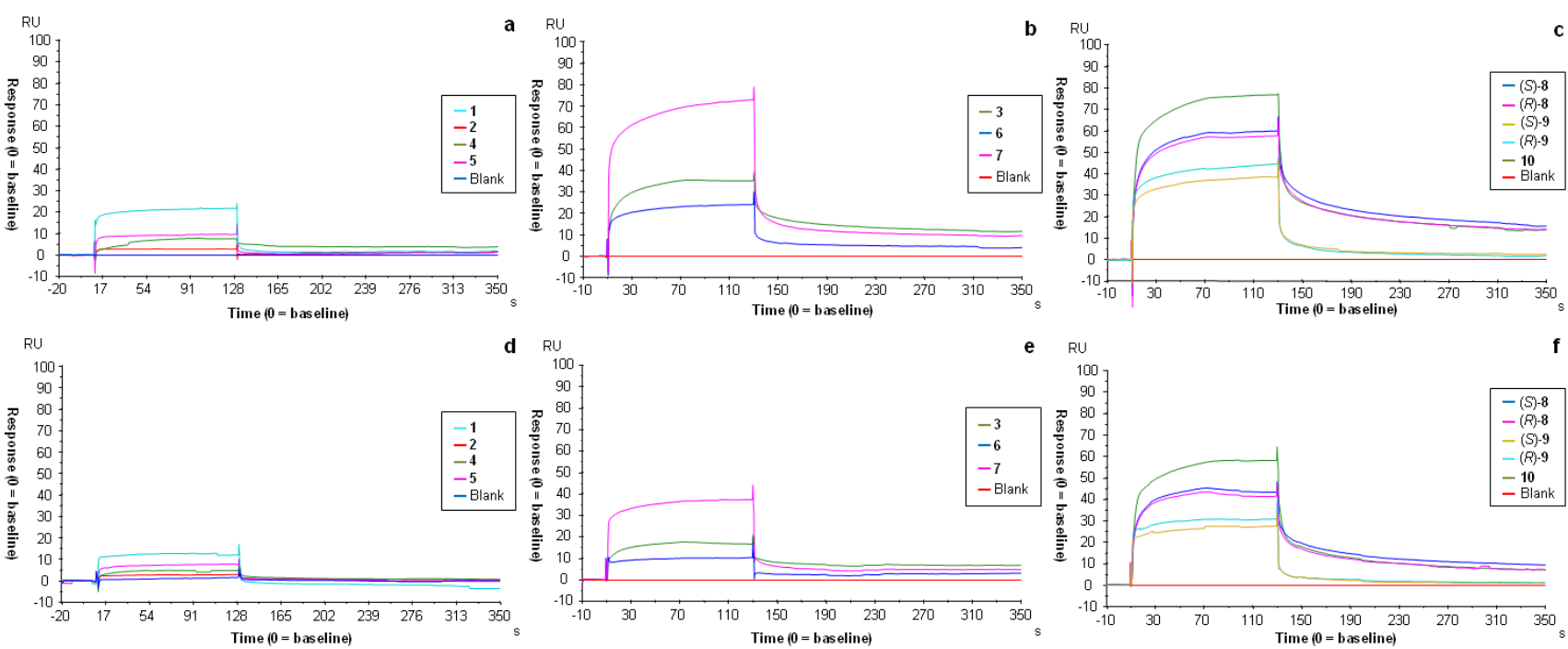

Figure 1. SPR analysis of the binding of compounds 1-10 to gp120 (a, b and c, chip density 3256 RU) and to gp41 (d, e and f, chip density 3703 RU) injected as a $5 \%$ DMSO solution over the surface at a fixed concentration of $50 \mu \mathrm{M}$. Running buffer was $\mathrm{HBS}-\mathrm{P}$ at $\mathrm{pH} 7.4$ containing $10 \mathrm{mM}$ of $\mathrm{Ca}^{2+}$.

Molecular Recognition Studies. The set of aminopyrrolic compounds was subjected to Surface Plasmon Resonance (SPR) analysis to determine their ability to interact with the highly mannosylated $\mathrm{N}$-glycans of the HIV viral envelope glycoproteins gp120 and gp41. From previous observations in highly competitive organic media (acetonitrile), the investigated molecules showed interesting binding affinities towards mannose glycosides (Table 1). While molecules $\mathbf{2}$ and $\mathbf{4}$ showed a negligible binding ability, the remaining receptors showed affinities ranging from high-millimolar $(98.0-44.5 \mathrm{mM})$ for 5 to low-millimolar (22-5.90 mM) for 1, 3, 6 and 7 to submillimolar (4 - $0.13 \mathrm{mM}$ ) for $(S)-\mathbf{8},(R)-\mathbf{8},(S)-\mathbf{9},(R)-\mathbf{9}$ and $\mathbf{1 0} .^{[11 \mathrm{~b}, 13]}$ The glycan recognition properties were thus investigated towards the glycoproteins gp120 and gp41 of the HIV envelope, covalently immobilized on a SPR sensor chip. Since recognition of these glycans by several CBAs of both peptidic and non-peptidic origin such as C-type lectins (e.g. MBL, DC-SIGN) and the nonpeptidic pradimicin antibiotics, is driven by a $\mathrm{Ca}^{2+}$-dependent carbohydrate binding, ${ }^{[9,14]}$ a $10 \mathrm{mM}$ concentration of calcium was used in the experimental set-up. To help the dissolution of the investigated molecules, $5 \% \mathrm{v} / \mathrm{v}$ DMSO was added to the HBS-P buffer solution at $\mathrm{pH} 7.4$.

In a first series of experiments (Figure 1), binding of the compounds to gp120 and gp41 was investigated, using high density gp120 (3,256 RU) and gp41 (3,703 RU) sensor chips. The compounds were injected at a relatively high concentration $(50 \mu \mathrm{M})$ to the HD chips to enable detection of potentially low binding affinities for the compounds. Interestingly, most of the investigated compounds showed a pronounced interaction with gp120 and gp41 (Figure 1). However, striking differences in terms of binding amplitude were observed among the set of compounds. Molecules 7 and 10 showed the strongest binding of the series for both glycoproteins (RU 75), followed by $(S)-8$, $(R)-8$ (RU between 50 and 75); an intermediate binding amplitude was observed for compounds 1, 3, 6, (S)-9 and (R)-9 (RU between 20 and 45). Finally, compounds 2, 4 and 5 showed the poorest $(R U \ll 20)$ interaction with both viral glycoproteins $(R U<20)$. Aspecific interactions were separately tested in a second set of experiments (Figure S1), in parallel with the gp120/gp41 interaction experiments, by examining the binding amplitude generated by the investigated compounds when exposed to a HSA (non-glycosylated human serum albumin) $(5,520 \mathrm{RU})$ sensorchip. In general, the order of binding magnitude of the compounds to gp120 and gp41 in this experiment was virtually identical to that of the previous experiment reported in Figure 1. Interestingly, all compounds presented a rather weak interaction with human serum albumin, with the exception of compound 10, which showed a pronounced binding amplitude ( $65 \mathrm{RU})$. Probably, the propensity to establish aspecific interactions with nonglycosylated proteins provides an additional (but not predominant) contribution to the binding abilities observed toward gp120 and gp41.

From the comparison of binding abilities observed via SPR through immobilized gp120 and gp41 with the data previously obtained in acetonitrile for mannoside interaction, a direct correlation became apparent. In particular, while the submillimolar affinities for mannosides measured for compounds $(S)-8,(R)-\mathbf{8},(S)-\mathbf{9}, \quad(R)-\mathbf{9}$ and $\mathbf{1 0}$ are reflected by a high interaction observed with gp120 and gp41, poor or negligible affinities of compounds $\mathbf{2}, \mathbf{4}$ and $\mathbf{5}$ are reflected by the absence of significant interactions with the glycoproteins. This evidence suggests that the interaction between the investigated compounds and the two glycoproteins is most likely driven by 
the molecular recognition of the high-mannose type glycans. In analogy to other non-peptidic CBAs, such as PRM-A and PRM$S$, these compounds may recognize the mannose residues, promoting their adhesion to the immobilized gp120 and gp41.

Cytostatic activity. The binding interaction observed via SPR suggested a potential role of the investigated molecules as CBAs and encouraged further investigation of their biological properties. The in vitro toxicity against $\mathrm{HuH}-7$ (Human Hepatoma) cells of compound $\mathbf{1}$ was reported in a previous paper. Thus, cytostatic activity of the whole set of molecules was investigated against human $\mathrm{CD}^{+}{ }^{+}$-lymphocyte CEM, murine leukemia L1210, and human cervix carcinoma HeLa cell lines (Table 2). The inhibitory effect

Table 2. Inhibitory effects of the compounds on the proliferation of murine leukemia (L1210) and human T-lymphocyte (CEM) and human cervix carcinoma (HeLa) cells.)

\begin{tabular}{llll}
\hline \multirow{2}{*}{ Compound } & \multicolumn{3}{c}{$I_{50}{ }^{a}(\mu \mathrm{M})$} \\
\cline { 2 - 4 } & $\mathrm{L} 1210$ & $\mathrm{CEM}$ & $\mathrm{HeLa}$ \\
\hline $\mathbf{2}$ & $0.91 \pm 0.17$ & $0.88 \pm 0.09$ & $0.94 \pm 0.21$ \\
$\mathbf{3}$ & $>250$ & $>250$ & $>250$ \\
$\mathbf{4}$ & $22 \pm 3$ & $19 \pm 3$ & $19 \pm 3$ \\
$\mathbf{5}$ & $17 \pm 3$ & $18 \pm 0$ & $21 \pm 3$ \\
$\mathbf{6}$ & $0.83 \pm 0.17$ & $1.2 \pm 0.3$ & $3.1 \pm 1.9$ \\
$\mathbf{7}$ & $88 \pm 38$ & $92 \pm 22$ & $138 \pm 60$ \\
$(S)-8$ & $58 \pm 30$ & $90 \pm 16$ & $123 \pm 60$ \\
$(R)-8$ & $9.6 \pm 6.3$ & $7.5 \pm 3.6$ & $9.4 \pm 1.7$ \\
$(S)-9$ & $8.6 \pm 5.4$ & $4.1 \pm 0.8$ & $4.6 \pm 1.3$ \\
$(R)-9$ & $0.70 \pm 0.18$ & $0.85 \pm 0.07$ & $0.77 \pm 0.05$ \\
$\mathbf{1 0}$ & $0.67 \pm 0.07$ & $0.82 \pm 0.02$ & $0.89 \pm 0.09$ \\
\hline & $89 \pm 18$ & $74 \pm 5$ & $24 \pm 4$ \\
\hline
\end{tabular}

[a] ${ }^{\mathrm{a}} 50 \%$ inhibitory concentration or compound concentration required to inhibit cell proliferation by $50 \%$.

measured showed a similar trend on the three different cell lines, whereas the antiproliferative activity markedly varied depending the nature and structure of compounds. Compounds containing the 1,3,5-triethylbenzene scaffold and featuring two or three aminopyrrolic binding arms $(\mathbf{1}, \mathbf{5})$ were markedly cytostatic. When the ethyl groups were removed from the scaffold of the parent molecule 1 , the resulting compound 3 exhibited a more than 20-fold decrease in cytostaticity. The tetra- and hexaaminopyrrolic compounds lacking ethyl groups were poorly cytostatic (i.e., $I C_{50}$ for compounds 6 and 7: $\sim 100$-fold higher than the $I C_{50}$ of compound 1). Thus, the specific substitution/conformation of the scaffold apparently plays a role in the cytostatic activity of these compounds. The presence of ethyl groups on the phenyl core, in addition to at least 2 or 3 aminopyrrolic arms, appears to be responsible for the appearance of cytostatic activity. However, it is noteworthy that compound 10, possessing an amino group in position 5 of pyrroles, unexpectedly lost its cytostatic activity compared to compound 1.

Compounds $(S)-\mathbf{8},(R)-\mathbf{8},(S)-\mathbf{9}$ and $(R)-\mathbf{9}$ represent further examples that show that the ethyl groups on the phenyl core are relevant for cytostatic activity. Moreover, compounds (S)-9 and $(R)-9$ (containing three pyrrolic side-arms) were more cytostatic than compounds $(S)-8$ and $(R)-8$ that contain only one pyrrole within the macrocyclic architecture. It was also interesting to note that the different ( $S$ and $R$ ) stereochemistry of the $\mathbf{8}$ and $\mathbf{9}$ compounds did not affect their cytostatic activities.

Antiviral Activity. The ability of the investigated compounds to protect CEM cells against the cytopathogenicity of HIV-1 and HIV-2 was evaluated to examine their antiviral activity (Table 3).

Table 3. Anti-HIV-1 and -HIV-2 activity of the compounds.

\begin{tabular}{lll}
\hline \multirow{2}{*}{ Compound } & \multicolumn{2}{c}{$E C_{50}{ }^{\mathrm{a}}(\mu \mathrm{M})$} \\
\cline { 2 - 3 } & $\mathrm{HIV}-1$ & $\mathrm{HIV}-2$ \\
\hline $\mathbf{2}$ & $>0.4$ & $>0.4$ \\
$\mathbf{3}$ & $>250$ & $>250$ \\
$\mathbf{5}$ & $>10$ & $>10$ \\
$\mathbf{6}$ & $>10$ & $>10$ \\
$\mathbf{7}$ & $>2$ & $>2$ \\
$(S)-8$ & $>50$ & $>50$ \\
$(R)-8$ & $16 \pm 0.0$ & $24 \pm 2.1$ \\
$(S)-9$ & $>2$ & $>2$ \\
$(R)-9$ & $>2$ & $>2$ \\
$\mathbf{1 0}$ & $>0.4$ & $>0.4$ \\
\hline
\end{tabular}

[a] ${ }^{a} 50 \%$ inhibitory concentration or compound concentration required to inhibit cell proliferation by $50 \%$.

Among the tested compounds $\mathbf{7}$ and $\mathbf{1 0}$ that showed the highest binding efficiency to gp120/gp41 exerted pronounced inhibitory effects against HIV infection. In particular, compound 10 showed the highest inhibitory activity against the HIV-1 infection $\left(E C_{50}\right.$ HIV-1: $1.7 \mu \mathrm{M}$ ), although it was markedly less active against HIV-2 $\left(E C_{50}: 50 \mu \mathrm{M}\right)$. Interestingly, compound 10 was poorly cytostatic. Apparently, there does not seem to exist an obvious correlation between gp120/gp41 binding and anti-HIV-1 activity in cell culture. This can be explained by considering that a number of compounds may bind to non-neutralizing parts of the gp120 glycoprotein, which do not affect the gp120/gp41-driven viral entry into the target cells. It should, however, also be noted that some of the gp120/gp41-binding compounds such as 8 and 
9 were fairly toxic to the cell cultures, thereby masking a potential antiviral activity. None of the compounds showed inhibitory activity against a broad panel of DNA and RNA viruses (Tables in Supporting Information), except for compound 7 that was found to be active against respiratory syncytial virus (RSV), showing an $E C_{50}$ of $4 \mu \mathrm{M}$.

\section{Conclusions}

In the present work a set of structurally related synthetic aminopyrrolic receptors for mannosides, endowed with antimycotic activities against yeast and yeast-like pathogens presenting on their cell wall high-mannose-type glycans, has been investigated to ascertain the ability to target the HIV highmannose-type glycans of the gp120 glycoprotein and to exert antiviral activity. Binding measurements towards gp120 and gp41, obtained by SPR, demonstrated a good correlation between mannose recognition and interaction with the glycoprotein. Moreover, compounds 7 and 10, which are the least cytostatic compounds of the set, demonstrated a significant inhibitory effect against HIV infection of T-lymphocyte CEM cells, suggesting that they possess antiviral activity like other CBAs, such as PRM-A and PRM-S. Thus, this new family of CBAs targeting gp120 and gp41, and showing anti-HIV activity, may be considered as potential novel anti-HIV lead candidates to be further developed.

\section{Acknowledgements}

The work was performed with financial support for the $\mathrm{KU}$ Leuven (GOA 10/14) and the Flemish FWO. We thank Mrs. Leen Ingles, Mrs. Lizette van Berckelaer, Mrs. Leentje Persoons and Mrs. Frieda De Meyer for dedicated technical assistance.

Keywords: CBAs $\cdot$ antiviral $\cdot$ mannose $\cdot$ HIV $\bullet$ synthetic receptors

[1] a) The Sugar Code: Fundamentals of Glycosciences (Ed.: H.-J. Gabius), Wiley-VCH, Weinheim, 2009; b) B. Ernst, W. Hart, P. Sinaÿ, Carbohydrates in Chemistry and Biology, Part I.2 and Part II.4, WileyVCH, Weinheim, 2000; c) T. K. Lindhorst, Essentials of Carbohydrate Chemistry and Biochemistry, 1st ed., Wiley-VCH, Weinheim, 2000.

[2] R. Medzhitov, C. A. Janeway, Jr., Science 2002, 296, 298-300.

[3] C. N. Scanlan, J. Offer, N. Zitzmann, R. A. Dwek, Nature 2007, 446, 1038-1045.

[4] a) J. Balzarini, Nat. Rev. Microbiol. 2007, 5, 583-597; b) J. Balzarini, Lancet Infect. Dis. 2005, 5, 726-731; c) J. Balzarini Antiviral Res. 2006 71, 237-247; d) K. O. Francois, J. Balzarini, Med. Res. Rev. 2012, 32, 349-387.

[5] a) S. Shahzad-ul-Hussan, E. Gustchina, R. Ghirlando, G. M. Clore, C. A. Bewley, J. Biol. Chem. 2011, 286, 20788 -20796; b) D. Huskens, G.
Férir, K. Vermeire, J.C. Kehr, J. Balzarini, E. Dittmann, D. Schols, J Biol Chem 2010, 285, 24845-24854

[6] a) J. Balzarini, K. Van Laethem, S. Hatse, K. Vermeire, E. De Clercq, W Peumans, E. Van Damme, A. M. Vandamme, A. Bolmstedt, D. Schols J. Virol. 2004, 78, 10617-10627; b) Q. Hu, N. Mahmood, R. J. Shattock. Virology 2007, 368, 145-154

[7] a) Y. Igarachi, T. Oki, Adv. Appl. Microbiol. 2004, 54, 147-166; b) F. Hiramoto, N. Nomura, T. Furumai , Y. Igarashi, T. Oki, Biosci. Biotechnol. Biochem. 2005, 69, 238-241.

[8] a) K.L. Oakley, C.B. Moore, D.W. Denning, Int. J. Antimicrob. Agents 1999, 12, 267-269; b) C.E. Gonzalez, A.H. Groll, N. Giri, D. Shetty, I. Al-Mohsen, T. Sein, E. Feuerstein, J. Bacher, S. Piscitelli, T.J. Walsh, Antimicrob. Agents Chemother. 1998, 42, 2399-2404.

[9] a) J. Balzarini, K. Van Laethem, D. Daelemans, S. Hatse, A. Bugatti, M. Rusnati,; Y. Igarashi, T. Oki, D. Schols, J. Virol. 2007, 81, 362-373; b) J. Balzarini, K. O. François, K. Van Laethem, B. Hoorelbeke, M. Renders, J. Auwerx, S. Liekens, T. Oki, Y. Igarashi, D. Schols, Antimicrob. Agents Chemother. 2010, 54, 1425-1435 c) C. A. Bewley, S. Shahzad-ul-Hussan, R. Ghirlando, C. I. Dogo-Isonagie, Y. Igarashi, J. Balzarini, C.A. Bewley, J Am Chem Soc 2012, 134, 12346-12349; d) V. Lozano, L. Aguado, B. Hoorelbeke, M. Renders, M. J. Camarasa, D. Schols, J. Balzarini, A. San-Félix, M. J. Pérez-Pérez, J Med Chem 2011, 54, 5335-5348; e) J. I. Jay, B.E. Lai, D.G. Myszka, A. Mahalingam, K. Langheinrich, D. F. Katz, P. F. Kiser, Mol Pharm 2010, 7, 116-129; f) A. Mahalingam, A. R. Geonnotti, J. Balzarini, P. F. Kiser, Mol Pharm 2011, 8, 2465-2475

[10] a) O. Francesconi, M. Gentili, C. Nativi, A. Ardá, F.J. Cañada, J. Jiménez-Barbero, S. Roelens, Chem. Eur. J. 2014, 20, 6081-6091; b) O Francesconi, M. Gentili, S. Roelens, J. Org. Chem. 2012, 77, 75487554 ; c) M. Cacciarini, C. Nativi, M. Norcini, S. Staderini, O. Francesconi, S. Roelens, Org. Biomol. Chem. 2011, 9, 1085-1091; d) C. Nativi, M. Cacciarini, O. Francesconi, A. Vacca, G. Moneti, A. lenco, S. Roelens, J. Am. Chem. Soc. 2007, 129, 4377-4385; e) O. Francesconi, A. lenco,; G. Moneti,; C. Nativi,; S. Roelens, Angew. Chem. Int. Ed. 2006, 43, 6693-6696.

[11] a) O. Francesconi, C. Nativi, G. Gabrielli, M. Gentili, M. Palchetti, B. Bonora, S. Roelens, Chem. Eur. J.. 2013, 19, 11742-11752; b) C. Nativi, O. Francesconi, G. Gabrielli, A. Vacca, S. Roelens, Chem. Eur. J. 2011, 17, 4814-4820; c) A. Ardá, F.J. Cañada, C. Nativi, O. Francesconi, G. Gabrielli,; A. lenco, J. Jiménez-Barbero, S. Roelens, Chem. Eur. J. 2011, 17, 4821-4829; d) A. Ardá, C. Venturi, C. Nativi, O. Francesconi, G. Gabrielli, F.J. Cañada, J. Jiménez-Barbero, S. Roelens, Chem. Eur. J. 2010, 16, 414-418; e) A. Ardá, C. Venturi, C. Nativi, O. Francesconi, F.J. Cañada, J. Jiménez-Barbero, S. Roelens, Eur. J. Org. Chem. 2010, (1), 64-71; f) C. Nativi, M. Cacciarini, O. Francesconi, G. Moneti, S. Roelens, Org. Lett. 2007, 9, 4685-4688.

[12] a) Y. Nakagawa, T. Doi, Y. Masuda, K. Takegoshi, Y. Igarashi, Y. Ito, J. Am. Chem. Soc. 2011, 133, 17485-17493; b) Y. Nakagawa, T. Doi, T. Taketani, K. Takegoshi, Y. Igarashi, Y. Ito, Chem. Eur. J. 2013, 19, 10516-10525.

[13] C. Nativi, O. Francesconi, G. Gabrielli, I. De Simone, B. Turchetti, T. Mello, L. Di Cesare Mannelli, C. Ghelardini, P. Buzzini, S. Roelens Chem. Eur. J. 2012, 18, 5064-5072.

[14] a) H. Feinberg, D. A. Mitchell, K. Drickamer, W. I. Weis, Science 2001, 294, 2163-2166; b) I. Botos, A. Wlodawer, Prog. Biophys. Mol. Biol 2005, 88, 233-282.

[15] A. Vacca, O. Francesconi, S. Roelens, Chem. Rec. 2012, 12, 544-566. 


\section{FULL PAPER}

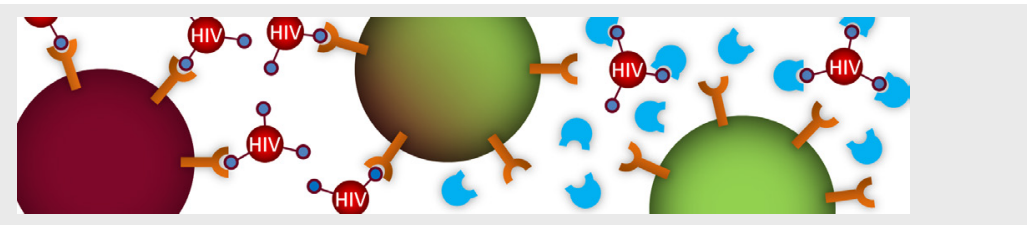

Aminopyrrolic synthetic receptors for mannosides, capable of interacting with the gp120 and gp41 glycoproteins of the HIV viral envelope, have demonstrated significant inhibitory activity against HIV infection of T-lymphocyte CEM cells, together with a poor cytostatic activity.
Oscar Francesconi, * Cristina Nativi,

Gabriele Gabrielli, Irene De Simone, Sam Noppen, Jan Balzarini, Sandra Liekens and Stefano Roelens

\section{Page No. - Page No.}

Antiviral Activity of Synthetic Aminopyrrolic Carbohydrate Binding Agents. Targeting the Glycans of Viral gp120 to Inhibit HIV Entry. 